\title{
Uniform faint reticulate pigment network - A dermoscopic hallmark of nevus depigmentosus
}

\section{Surit Malakar ${ }^{1}$, Samipa Samir Mukherjee ${ }^{2,3}$, Subrata Malakar ${ }^{3}$}

\author{
${ }^{1} 1^{\text {st }}$ Year Post graduate, Department of Dermatology, SUM Hospital Bhubaneshwar, India, ${ }^{2}$ Department of Dermatology, \\ Cloud nine Hospital, Bangalore, India, ${ }^{3}$ Department of Dermatology, Rita Skin Foundation, Kolkata, India
}

Corresponding author: Dr. Samipa Samir Mukherjee, E-mail: drsamipamukherjee@gmail.com

Sir,

Nevus depigmentosus (ND) is a localized hypopigmentation which most of the time is congenital and not uncommonly a diagnostic challenge. ND lesions are sometimes difficult to differentiate from other hypopigmented lesions like vitiligo, ash leaf macules and nevus anemicus. Among these naevus depigmentosus poses maximum difficulty in differentiating from ash leaf macules because of clinical as well as histological similarities [1]. Although the evolution of newer diagnostic techniques like dermoscopy has obviated the need for invasive diagnostic procedure, evidence and literature is yet to build on different hypopigmentary conditions. We herein describe the dermoscopic features of nevus depigmentosus and rightfully compare it with the features of its close contenders namely vitiligo and ash leaf macules to establish its distinguishing dermoscopic patterns.

A 9 month old male child, born of non consanguineous marriage, presented with a white patch on the abdomen since birth. The mother had brought the patient for evaluation owing to the cosmetic concerns. History and physical examination of the child was non contributory. Local examination of the skin revealed a well defined hypo- depigmented macule with no evidence of leucotrichia located on the left side of the abdomen $1.5 \mathrm{~cm}$ away from the umbilicus. Diascopy test was negative and revealed feathering of margins and accentuation of the hypopigmentation. Wood's lamp evaluation showed an off white color. We went on to do the dermoscopy of the lesion to establish its pattern. Dermoscopy revealed uniform faint reticular melanocytic/pigment network throughout the lesion without any evidence of total loss of pigmentary network (Fig. 1).
ND is a form of cutaneous mosaicism with functionally defective melanocytes and abnormal melanosomes. Histopathologic examination shows normal to decreased number of melanocytes with S-100 stain and less reactivity with 3,4-dihydroxyphenylalanine reaction and no melanin incontinence [2]. Electron microscopic findings show stubby dendrites of melanocytes containing autophagosomes with aggregates of melanosomes.

For ease of understanding the pigmentary network patterns will be compared with that of normal skin will be provided herewith (Fig. 2). Normal reticulate pattern of pigmentation seen over normal skin corresponds to the pigmentation of the keratinocytes along the rete ridges while the pale area in between corresponds to the papillary dermis [3].

Vitiligo is an autoimmune disease of unknown etiology characterized by destruction and absence of melanocytes. Pigmentary network changes, and perifollicular and perilesional hyperpigmentation on polarized light examination, and a diffuse white glow on ultraviolet light examination were noted in evolving vitiligo lesions [4]. Established lesions of vitiligo demonstrate complete absence of reticulate pigmentary network as opposed to the normal skin which corresponds to the destruction of melanocyte and absence of melanin histologically (Fig. 3a).

Ash leaf spots (ALS) form an important part of the diagnostic criteria of tuberous sclerosis complex (TSC) although less than 2 ALS can be seen in normal individuals without TSC. On dermoscopy ash leaf macules are characterized by areas of faint reticular

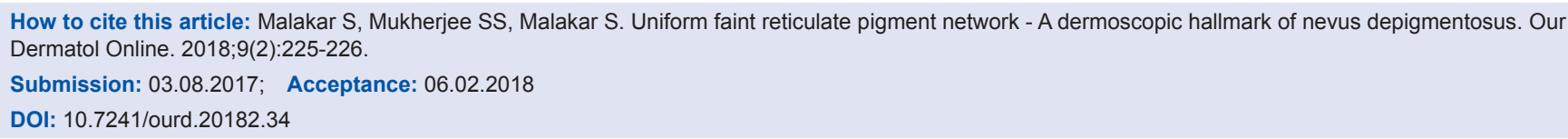




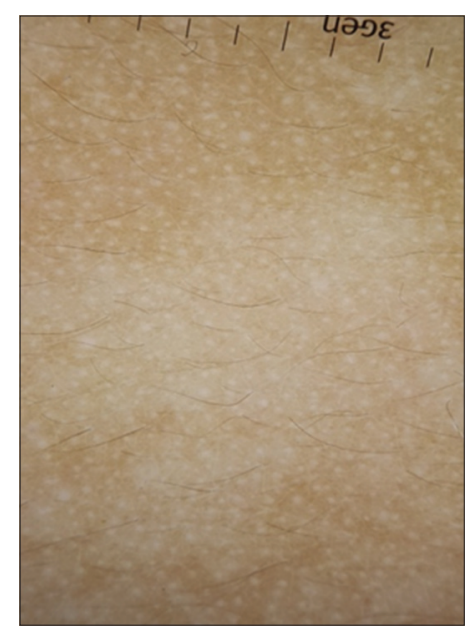

Figure 1: Nevus depigmentosus showing uniform faint reticulate pigmentary network pattern.

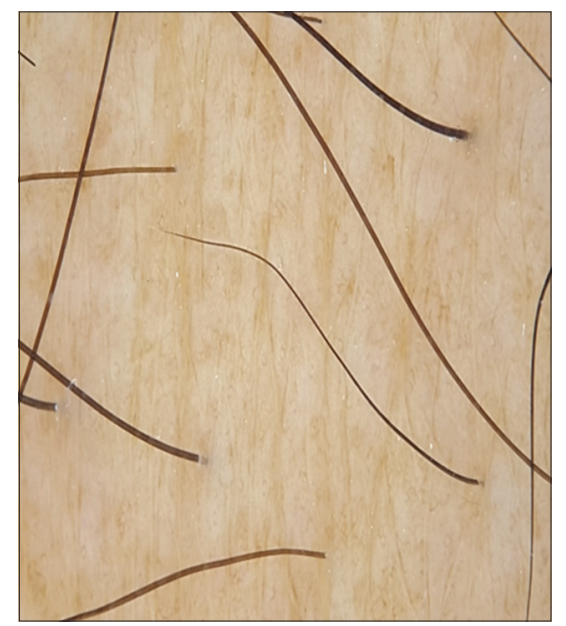

Figure 2: Normal skin showing reticulate pigmentary network (for comparison).

pigmentation and zones of total loss of any pigmentary pattern (Fig. 3b).

Hypopigmentation in ND is due to defect in transfer of melanosomes from melanocytes to keratinocytes rather than total loss of pigment [5]. Melanocytes usually are normal in number and size. Melanosomes are usually normal in size, shape, and internal structure, but can be diminished in number, heteromorphic, aggregated in melanocytes, or located in membrane bound aggregates [6]. This pathology manifests itself dermoscopically as the presence of faint reticular pigment pattern as opposed to total loss of pigment(due to absent melanocytes) as in vitiligo (Fig. 1).

To summarise, dermoscopy forms an essential non invasive diagnostic tool for hypopigmented lesions.

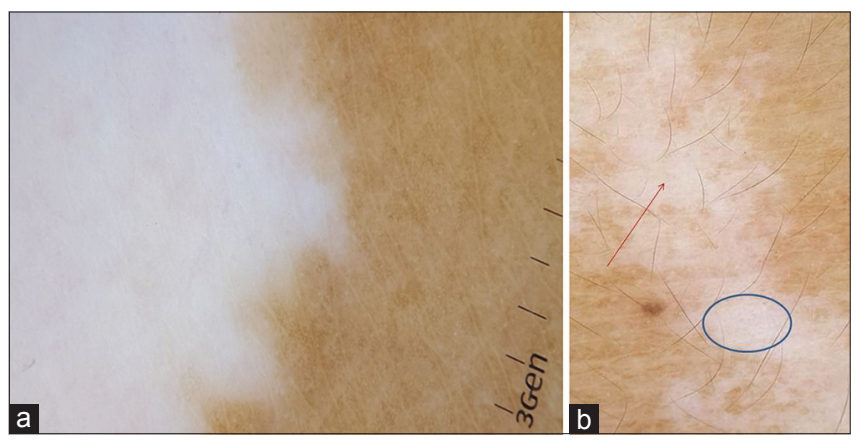

Figure 3: (a) Vitiligo showing loss of reticulate pigment network. (b) Ash leaf spots showing areas of faint reticular pigmentation and zones of total loss of any pigmentary pattern.

Differentiation of Nevus Anemicus from Nevus Depigmentosus is done by diascopy and firmly stroking lesional area. Vitiligo shows absence of melanocytic network.

Nevus Depigmentosus reveals uniform faint reticular pigment pattern whereas faint melanocytic network interspersed with total loss of the network characterizes ALS dermoscopically. To the best of our knowledge this is the first ever description of dermoscopic findings in nevus depigmentosus in the skin of color.

\section{REFERENCES}

1. Jindal R, Jain A, Gupta A, Shirazi N. Ash-leaf spots or naevus depigmentosus: a diagnostic challenge. BMJ Case Reports. 2013;2013:bcr2012007008.

2. Kim SK, Kang HY, Lee ES, Kim YC. Clinical and histopathologic characteristics of nevus depigmentosus. J Am Acad Dermatol. 2006;55:423-8.

3. Haldar SS, Nischal KC, Khopkar US. Dermoscopy: Applications and patterns in diseases of the brown skin. In: Uday K, editor. Dermoscopy and Trichoscopy in Diseases of the Brown Skin: Atlas and Short Text. New Delhi, India: Jaypee Brothers Medical Publishers; 2012.

4. Thatte SS, Khopkar US. The utility of dermoscopy in the diagnosis of evolving lesions of vitiligo. Indian J Dermatol Venereol Leprol. 2014;80:505-8.

5. Lee HS, Chun YS, Hann SK. Nevus depigmentosus: Clinical features and histopathologic characteristics in 67 patients. J Am Acad Dermatol. 1999;40:21-6.

6. Shim JH, Seo SJ, Song KY, Hong CK. Development of multiple pigmented nevi within segmental nevus depigmentosus. J Korean Med Sci. 2002;17:133-6.

Copyright by Surit Malakar, et al. This is an open-access article distributed under the terms of the Creative Commons Attribution License, which permits unrestricted use, distribution, and reproduction in any medium, provided the original author and source are credited.

Source of Support: Nil, Conflict of Interest: None declared. 